95. Deutscher Röntgenkongress /7. Gemeinsamer Kongress von DRG und ÖRG

\section{Einladung zur Mitgliederversammlung der DRG am Donnerstag, den 29. Mai 2014}

Zur ordentlichen Mitgliederversammlung der Deutschen Röntgengesellschaft, Gesellschaft für Medizinische Radiologie e. V (DRG), am Donnerstag, den 29. Mai 2014, von 17:15 - 18:45 Uhr im Congress-Centrum-Hamburg $(\mathrm{CCH})$ im Saal Werner, lädt der Vorstand die Mitglieder der DRG hiermit herzlich ein.

\section{Tagesordnung}

$\nabla$

- TOP 1 Begrüßung

- TOP 2 Totengedenken

- TOP 3 Genehmigung des Protokolls der Mitgliederversammlung vom 30. Mai 2013 (veröffentlicht im Jahresbericht 62, Januar 2014)
- TOP 4 Bericht des Präsidenten

- TOP 5 Bericht des Schatzmeisters

- TOP 6 Bericht der Kassenprüfer

- TOP 7 Entlastung des Vorstandes

- TOP 8 Vorschläge für Ehrungen 2015

- TOP 9 Wahl der Kassenprüfer 2015

- TOP 10 Wahl des Kongresspräsidenten 2017

- TOP 11 Erhöhung der Mitgliedsbeiträge

> TOP 12 Einladung zum Röntgenkongress 2015

- TOP 13 Bericht der Akademie für Fort- und Weiterbildung in der Radiologie Verleihung der Wachsmann-Preise und des Promotionspreises
- TOP 14 Bericht der Vorsitzenden der Vereinigung der Medizinisch-Technischen Berufe in der DRG (VMTB)

- TOP 15 Bericht des Justitiars der DRG

- TOP 16 Verschiedenes

Die Einlasskontrolle erfolgt über den Kongressausweis. Entsprechend der Satzung der DRG können an der Mitgliederversammlung nur Mitglieder teilnehmen, deren Beitragskonto für 2014 ausgeglichen ist. Das Protokoll der Mitgliederversammlung 2013 sowie weitere relevante Dokumente finden Sie im DRG LOGIN-Bereich der DRG-Homepage (www.drg.de). Weitere Dokumente werden in Vorbereitung auf die Mitgliederversammlung 2014 im Mitgliederbereich der DRG-Homepage eingestellt.

Im Namen des Vorstandes

Prof. Dr. Norbert Hosten (Präsident) 